Aw. Chil. Pediatr. 63 (4); 205-208, 1992

\title{
Constipación crónica idiopática. Estudio longitudinal en cuarenta casos
}

\author{
Francisco Larraín B. ${ }^{1}$; Osvaldo Danús $V .1$ \\ Chronic constipation
}

\begin{abstract}
Chronic constipation is a common condition in childhood, and despite current diagnostic methods its etiology remains unknown in $70 \%$ to $80 \%$ of the cases. Apropriate medical treatment is frequently effective but it has to be sustained and carefulty done in order to restore intestinal function. Data from 40 children (21 girls) with chronic idiopathic constipation who were seen along a 10 vears period at a pediatric gastroenterology clinic from a university associated hospital at Santiago, Chile, is shown. Symptoms begun betore one year of age in $62 \%$ and before age 3 vears in $82 \%$ of these patients. Sixty percent of them needed frequent use of laxatives, one third looked for medical care because of fecal runoff and $22.5 \%$ had psychological symptoms. A cross sectional study after one year follow up in 36 remaining patients showed that $21(61.7 \%)$ of them were free of symptoms and apparently cured. After ten years it was possible to regain information from 16 patients, 14 of them were considered to be healthy and their $x$-ray studies were normal. The two remeining cases showed clinical, radiological and manometric evidence of an organic disease (mvogenic achalasia, megarectus and megacolon) and both required surgical treatment. Possible causes of protracted course in children with chronic idiopathic constipation include genetic predisposition, failure to comply with treatment and chronic local inflamation and fibrosis due to persistent fecal mass.
\end{abstract}

IKey words: Constipation, chronic, idiopatic. treatment, prognosis.|

La constipación crónica es un problema frecuente en la infancia. En los casos graves, cuando se acompaña de fecaloma y escurrimiento fecal.

1. Unidad de Gastroenterolog ja, Departamento de Pediatr ia, Hospital Roberto del Río. constituye una situación muy molesta para el niño y la familia. En 70 a $80 \%$ de los pacjentes, los métodos radiológicos, manométricos e histológicos de estudio disponibles actualmente, no permiten demostrar alteraciones orgánicas y tampoco existen alteraciones de los hábitos de defecación o de alimentación que expliquen el 
trastorno. Al no poder atribuir el estreñiniento a ningún factor conocido hasta ahora, estos pacientes quedan catalogados como portadores de constipación crónica idiopática ${ }^{1}$. Pocos estudios de seguimiento evalúan la respuesta de esta afección, a largo plazo, al tratamiento médico ${ }^{2}$. En la práctica clínica es frecuente que, pese a manejos bien hechos, los pacientes sufran recaídas, se reproduzca el fecaloma y se desarrollen megarrecto y megacolon, que ensombrecen el pronóstico y desalientan al paciente y a su familia. Estos hechos nos motivaron a estudiar la evolución de un grupo de niños con el problema, que han sido atendidos en un hospital pediátrico de Santiago a lo largo de un período de 10 años.

\section{Material y Método}

Se revisaton las fichas clínicas de 40 niños con constipactión crónica idiopática, atendidos en la policlínica de gastroenterología del Hospital Roberto del Río, entre los arios 1978 y 1988 . En todos ellos, un estudio clínico completo (sintomas clínicos, examen físico, radiolog ia y manometría) descartó las causas conocidas de constipación.

La edad de los pacientes en la primera consulta variaba entre 6 meses $y, 11$ arios; 5 eran menores de 2 años; 21 tenian 2 a 5 años; 14 tenían más de 5 años y 21 de los 40 eran mujeres.

El grado de constipación al comienzo del estudio fue clasificado, de acuerdo a un trabajo anterior, como leve (grado I), cuando cedía con facilidad a un régimen alimentario adecuado, sin necesidad de usar medicanentos o enemas evacugntes; moderado (grado II), si el paciente requeria laxantes en forma rutinaria para obrar, y severo (grado UI) cuando se obtenían evacuaciones sólo mediante el uso de lavados intestinales repetidos ${ }^{5}$.

Una vez confirmado el diagnóstico se explicó a los padres las caracteristicas de la enfermedad y se inició un tratamiento médico, que consistió, en primer hugar, en lavados intestinales, hasta obtener el vaciamiento del recto, para lo cual -ocasionalmente- el paciente de bió ser hospitalizado para evacuar el fecalome. Sinnultáneamente con los lavados se indicó un régimen rico en residuos, lubricantes (vaselina líquida) en los mayores de tres anos o ablandadores de la deposición (dioctil sulfosuccinato sódico) en los nitios menores, en cantidad suficiente para lograr evacusaciones fáciles. Se realizó también una educación del paciente y de los padres, para fomarle un hábito intestinal normal. Este tratamiento se mantuvo por lo menos un ano, indicándose controles posteriores cada 6 meses para prevenir recaidas.

El resultado del tratamiento a corto plazo se estudío de las fichas clínicas, analizando los síntomas al año de tratamiento. El estado actual de los pacientes se evaluó mediante visita de la asistente social del hospital y citación a control médico radiológico de los pacientes. Sólo 16 de los 40 pactentes del grupo inicial pudieron ser ubicados, después de lo cual fueron controlađos en el hospital, completándose sus registros clínicos con la información que faltaba desde la última consulta.

\section{Resultados}

La magnitud de la constipación de los pacientes en la primera consulta era en $4(10 \%)$ grado I, en $24(60 \%)$ grado Il y en $12(30 \%)$ grado III. Los sintomas comenzaron precozmente, en el primer año de vida, en $25(62 \%)$ de los casos y antes de la edad escolar en 90\% de ellos (tabla 1). Los síntomas y signos más relevantes que presentaron estos pacientes se describen en la tabla 2. Entre ellos destacaron: distensión abdominal, escurrimiento fecal $y$ la rectorragia secundaria a fisura ana. Estos dos últimos constituyeron casi siempre el motivo principal que alarmó a los padres y los hizo solicitar una consulta para el especialista. El escurrimiento fecal fue muchas veces interpretado en forma errónea como diarrea. Nueve pacientes $(22,5 \%)$ tenían trastomos psicológicos. En el examen físico se comprobó la presencia de fecaloma en la gran mayoría de los pacientes, lo que revelaba el largo tiempo de enfermedad.

Para analizar la evolución clínica y la respues. ta al tratamiento es necesario considerar dos etapas en el estudio. Después de un año de tratamiento, 34 nínos contínuaban en control y en $21(61,7 \%)$ había desaparecido la constipación, no habiendo tenido necesidad de usar medicamentos ni enemas en los últimos 6 meses. Trece pacientes seguían con constipación, que en tres era grave, con reinstalación del fecaloma, por lo cual fueron reevaluados con radiología

Tabla L

Constipación crónica idiopática. Edad de comienzo de la constipación ( 40 casos)

\begin{tabular}{ccr}
\hline Edad (años) & n niños & $\%$ \\
\hline$<1$ & 25 & 62,0 \\
$1-2$ & 2 & 5,0 \\
$2-3$ & 6 & 15,0 \\
$3-4$ & 1 & 2,5 \\
$4-5$ & 2 & 5,0 \\
$>5$ & 3 & 7,5 \\
No precisada & 1 & 2,5 \\
\hline
\end{tabular}


Tabla 2

Constipación crónica idiopática. Manifestaciones clinicas $y$ complicaciones ( 40 casos)

- $n$

\section{SINTOMAS}

Rectorragia

Distensión abdominal

Escurrimiento fecal

Trastornos psicológicos

Dolor abdominal

Infección urinaria

Prolapso rectal

37,5

35,0

32,5

22,5

15,0

12,5

12,5

SIGNOS

Fecaloma

Masa abdominal

31

Desnutrición
77.5

50,0

5.0 y manometria: uno tenja alteraciones manométricas compatibles con acalasia miogénica, debiendo ser sometido a esfinteromiomectomia; otro tenía el sigmoides redundante y muy dilatado, por lo que se le resecó el asa sigmoidea; ambos tuvieron buena evolución posterior.

A los 10 años sỏlo fueron ubicados 16 niños. Catorce habían mejorado, pues en el momento del control tenían deposiciones normales, cada 24 a $48 \mathrm{~h}$, sin necesidad de medicamentos ni lavados, $y$ el enema baritado era normal en todos ellos. Los otros dos niños continuaban con constipación, que era moderada en uno y grave en el otro. En ambos casos el enema baritado mostro megarrecto y megacolon $y$ en el estudio manométrico anorrectal habia alteraciones compatibles con acalasia miogénica, por lo que fueron sometidos a esfinteromiomectomía, con buenos resultados inmediatos.

\section{Discusión}

Los niños con constipación crónica y escurri. miento fecal presentaban habitualmente diversos trastornos psicológicos. Este hecho hizo pensar hace algún tiempo a algunos autores, que si se descartaban las diferentes causas orgánicas, la constipación podia atribuirse a estos trastornos y llamaron a esta afección "constipación psicogénica" . Trabajos recientes han demostrado que las alteraciones psiquicas desaparecen, en la mayoría de los casos, con el tratamiento de la cons- tipación $y$, por lo tanto, parecen ser consecuencia de ésta y no su causa. En la actualidad se prefiere llamar a esta afección "constipación crónica idjopática"1. En los últimos 15 años se han realizado numerosas investigaciones, en adultos y niños, tratando de identificar el trastorno básico de esta afección, demostrando la presencia de alteraciones de la motilidad y disminución de la velocidad del tránsito en distintos segmentos del colon. En base a estos hallazgos se ha clasificado a los pacientes en diferentes grupos, según la región afectada, pero no se ha avanzado más en el conocimiento de la etiopatogenia de la enfermedad?

Como en otras experiencias ${ }^{4}$, en estos pacientes los sintomas se iniciaron muy precozmente, antes del año de edad en la mayoría de los casos. La iniciación de la constipación, antes de que pudieran haberse alterado los hábitos de alimentación o de defecación, sumado a informes sobre antecedentes familiares de constipación en más de la mitad de los casos de otras series, hacen pensar en la existencia de predisposición genética para esta afección, sospecha apoyada por la concordancia seis veces mayor de constipación en gemelos univitelinos que en bivitelinos ${ }^{3}$.

Al año de tratamiento algunos enfermos pre. sentaban respuesta parcial o ninguna a pesar de cumplir las indicaciones y en unos pocos de ellos se encontró evidencia de acalasia miogénica. Este último trastorno se encuentra con cierta frecuencia en pacientes con constipación crónica $^{\text {" }}$, y es una complicación que ocurre a largo plazo, cuando el tratamiento de la constipación no es bien llevado, pues al persistir la retención fecal por períodos prolongados, la masa fecal se endurece, se impacta y provoca un proceso inflamatorio crónico en las paredes de] colon, recto y ano. En casos de inflamación muy intensa se desarrolla fibrosis, que puede comprometer el esfínter interno impidiendo su relajación cuando se genera el reflejo rectoanal. De esta manera se agrega un factor orgánico que agrava la constipación y la hace aún más rebelde al tratamiento, haciéndose necesario practicar esfinteromiomectomía. Esta complicación no se produce cuando se cumple bien el tratamiento y se evita la retención fecal. En estos casos, la evohución es buena, a corto y largo plazo, como pudo apreciarse en la mayoría de nuestros pacientes.

En el períado de estudjo de estos pacientes no se contó con técnicas actuales que permi- 
tieran descartas otras afecciones, como la displasia neuronal, que en algunas revisiones se presenta con una frecuencia similar a la de acalasia miogénica $^{s, 9}$.

\section{Resumen}

La constipación crónica es una patologia frecuente en el nif́o $y$ en un alto porcentaje (70 a $80 \%$ ) los procedimientos diagnósticos actuales no permiten identificar la etiología. En esta situación el tratamiento médico debe ser prolongado y tiende a rehabilitar la función intestinal. En este trabajo se presenta el resultado obtenido a 10 afios plazo, en un grupo de 40 nifos portadores de constipación crónica idiopática. Un corte realizado a un año de evolución demostró que $61,7 \%$ de los casos había mejorado. A los 10 años existió mejoría en $14 \mathrm{de}$ los 16 pacientes. En éstos el estudio radiológico de control fue normal. En los dos restantes, tanto el control radiológico como manométrico anorrectal, evidenciaron signos compatibles con alteraciones orgánicas (acalasia miogénica-megarrecto y megacolon) que indujeron al tratamiento quirúrgico. Las causas que pudieran condicionar la evolución tórpida en pacientes con constipación crónica idiopática incluyen, probablemente, factores genéticos, inflamación crónica y fibrosis de las paredes de colon distal, recto y ano, secundarjas a la persistencia de una masa fecal.

(Palabras claves: Constipación crónica, pronóstico.)

\section{Referencias}

1. Silverberg $M$, Daum $F$ : Textbook of Pedistric Gastroenterology, 2a, ed. Chicago: Year Book Med Publ 1988. Chapter 9.

2. Davidson $M$, Kugler MM, Bauer Ch: Diagnosis and Management in Children with Severe and Protacted Contstipation and Obstipation J Pedjatr 1963; 62: 261-275.

3. Bellman $M$ : Studies on Encopresis. Acta Paediat Scand (supp)) 1966; 170:1-150.

4. Abrahamian F, Lloyd-Still $J D$ : Chronic Constipation in Childhood. A Longitudinal Study of 186 Patients. J Pedjatr Gastroenterol Nutr 1984; 3: 460-467.

5. Larraín $F$, Aldunate $G$, Danús $O$ : Constipación Crónica y Megacolon. Estudio longitudinal en 106 niños. Rev Chil Pediatr 1975; 46: 405-410.

6. Anderson Ch, Burcke V: Paediatric Gastroente rology. London: Blackwell Scientific Publication, 1975. Chapter 14.

7. Hatch $T$ : Encopresis and Constipation in Children. Pediatr Clin North Am 1988; 35: 257-280.

8. Krebs $C$, Flores $G$, Bravo I, Cruz MA, Sepulveda R. Rojas $S$ : Electromanometría Anorrectosigmoidea en el diagnóstico diferencial de la constipación en el niño. Rev Chil Pediatr 1988; 69: 11-16.

9. Krebs C. Silva C, Porra MA: Anorectal electroma. nometry in the diagnosis of neuronal intestinal dysplasia in childhood. Eur J Pediatr Surg 1991; $1: 40-44$.

La Dirección de la Escuela de Postgrado de la Facultad de Medicina de la Universidad de Chile informa a los médicos egresados de los programas de formación de especialistas que no han rendido el examen final correspondiente, que se han fijado los siguientes plazos para cumplir ese requisito:

1. Años 1992 y 1993 , peŕ́odo de blanqueo de la situación para egresados desde 1983 a la fecha.

2. Hasta dos años después de terminado el programa para quienes egresen a partir de 1992 (D.U. 00966). 